\title{
Présences audibles et écoute en présence : pour une poétique sonore du théâtre
}

Audible presences and listening in presence: for a sonic poetics of the theatre

\section{Marcus Borja}

\section{(2) OpenEdition}

\section{Journals}

Édition électronique

URL : https://journals.openedition.org/rsl/1256

DOI : $10.4000 /$ rsl. 1256

ISSN : 2271-6246

Éditeur

Éditions Rue d'Ulm

\section{Référence électronique}

Marcus Borja, "Présences audibles et écoute en présence : pour une poétique sonore du théâtre », Revue Sciences/Lettres [En ligne], 5 | 2017, mis en ligne le 02 octobre 2017, consulté le 30 juin 2021. URL : http://journals.openedition.org/rsl/1256 ; DOI : https://doi.org/10.4000/rsl.1256

Ce document a été généré automatiquement le 30 juin 2021

(C) Revue Sciences/Lettres 


\title{
Présences audibles et écoute en présence : pour une poétique sonore du théâtre
}

Audible presences and listening in presence: for a sonic poetics of the theatre

\author{
Marcus Borja
}

Élément le plus subtil et le plus malléable du concret, le son n'a-t-il pas constitué, ne constitue-

t-il pas encore, dans le devenir de l'humanité comme dans celui de l'individu, le lieu de rencontre initial entre l'univers et l'intelligible?

Paul Zumthor ${ }^{1}$

1 "Théâtre ", nous le savons, vient du latin theatrum qui vient à son tour de l'ancien grec $\vartheta \varepsilon ́ \alpha \tau \rho o v$ (theatron), combinaison des morphèmes $\theta \varepsilon \alpha$ (voir) et $\tau \rho o v$ (dénotant un endroit). Le théâtre est donc le lieu où (et d'où) l'on voit. Il est intéressant de se rappeler aussi qu'autrefois, l'emplacement des bancs disposés de part et d'autre de la scène, à cour et à jardin, pour les gens de qualité - qui y restèrent jusqu'en 1759 - étaient aussi appelés « théâtre " ${ }^{2}$ lieu d'où l'on voit mais aussi, finalement, d'où l'on est vu.

2 En effet, la notion de contemplation, observation, regard (du côté du spectateur) posé sur une action (du côté de l'acteur) a toujours été, pour le meilleur comme pour le pire, associée au phénomène théâtral, l'élément visuel étant souvent la condition même pour que ce phénomène soit perçu comme tel. Peter Brook a écrit : «Je peux prendre n'importe quel espace vide et l'appeler une scène. Quelqu'un traverse cet espace vide pendant que quelqu'un d'autre l'observe, et c'est suffisant pour que l'acte théatral soit

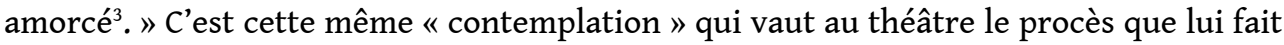
Platon par la bouche de Socrate dans le dernier livre de La République, et les « secours " divers que, selon Jacques Rancière, entendent lui prêter les avant-gardes théâtrales du $\mathrm{xx}^{\mathrm{e}}$ siècle, notamment le théâtre épique de Bertolt Brecht et le théatre de la cruauté d'Antonin Artaud. Dans Le Spectateur émancipé, Rancière parle, pour définir le théâtre en termes platoniciens, d'une «machine optique qui forme les regards à l'illusion et à la 
passivitét ». Et c'est toujours en termes d'optique qu'il résume les thèses théâtrales de l'Allemand et du Français :

Pour l'un il doit affiner son regard, pour l'autre il doit abdiquer la position même du regardeur. Les entreprises modernes de réforme du théâtre ont constamment oscillé entre ces deux pôles de l'enquête distante et de la participation vitale, quitte à mêler leurs principes et leurs effets 5 .

3 Mais jusqu'à quel point le phénomène théâtral est-il tributaire du phénomène visuel ? La présence scénique de la présence visible ? Jusqu'à quel point l'élément acoustique est-il le prolongement illustratif, confirmatif de l'élément visuel ? Peut-on aborder la scène uniquement par sa dimension sonore, ou du moins considérer cette dimension sonore en amont de tout préalable visuel?

4 C'est l'une des interrogations principales du projet de recherche artistique « Poétiques de la voix et espaces sonores", que je mène depuis 2014 au Conservatoire national supérieur d'art dramatique dans le cadre du programme doctoral d'art et de création SACRe (Science, Art, Création, Recherche) de l'École normale supérieure et de Paris Sciences et Lettres. Il s'agit d'une recherche pluridisciplinaire, au carrefour de la théorie et des pratiques artistiques, qui appelle, par sa nature et ses multiples résonances, une variation et une alternance de ses supports et de son outillage. Ainsi, plutôt que de hiérarchiser ou de délimiter les espaces plus ou moins étanches de la "théorie » et de la "pratique », la théorie, ici, ne saurait se concevoir autrement que comme structuration et relativisation d'une pratique concrète, et la pratique comme une mise en mouvement d'abstractions théoriques, en croisement et même en choc entre elles.

5 Nous nous efforçons de penser et de développer une pratique du théâtre à partir de l'écoute et du phénomène sonore, aussi bien en ce qui concerne la représentation que la composition dramaturgique. Cette dernière ne serait pas simplement la transposition en sons d'une forme dramatique donnée, comme une pièce radiophonique au sens " classique ", mais un agencement de signes sonores indépendamment d'un quelconque compromis diégétique préalable. Un équilibrage délicat, dans l'espace et dans la durée, de fragments sonores, timbres, rythmes, textures, dynamiques et enchaînements harmoniques (au sens large) au service d'une dramaturgie sensorielle et transdiégétique dont la signification se situerait en deçà et au-delà de toute dramaticité immédiate ${ }^{6}$.

[...] si les sons abandonnent leurs caractères représentatif et narratif pour travailler dans leurs flux abstraits, par leurs densités et leurs matérialités, toute question temporelle est résolue. L'abstraction sonore s'adapte à tous les temps, la durée de l'abstrait est sans échelle, sans mesure ${ }^{7}$.

Je tenterai ici de problématiser cette question de l'existence sonore du théâtre, en la mettant en perspective avec la première création scénique issue de cette recherche: Théâtre, spectacle sonore et choral pour cinquante acteurs en trente-cinq langues, créé en avril 2015 et repris en 2016 dans divers festivals ${ }^{8}$. Quatre documents audio en MP3, extraits de la captation qui a été faite de la représentation du 4 mars 2016 donnée au CNSAD, sont proposés en prolongement de lecture à la fin de ce texte. 


\section{Jouer par et pour l'oreille}

7 Il ne s'agit pas - il convient de le préciser - de faire le procès du visuel ou d'entretenir, en inversant les pôles, l'idée d'une quelconque hiérarchie sensorielle : ce serait inutile et inévitablement voué à l'échec ${ }^{9}$. Il s'agit, au contraire, d'aller " voir » ailleurs que dans une hiérarchie déjà bien établie qui assure au visuel la place prépondérante dans l'assimilation, l'appropriation et surtout l'interprétation, c'est-à-dire la confirmation herméneutique des expériences du monde sensible. Dans un très bel ouvrage consacré précisément à la question de l'audible, Jean-Luc Nancy écrit :

Figure et idée, théâtre et théorie, spectacle et spéculation se conviennent mieux, se superposent, voire se substituent avec plus de convenance que ne le peuvent l'audible et l'intelligible ou le sonore et le logique. Il y aurait, au moins tendanciellement, plus d'isomorphisme entre le visuel et le conceptuel, ne serait-ce qu'en vertu de ceci que le morphé, la «forme» impliquée dans l'idée d'« isomorphisme », est d'emblée pensée ou saisie dans l'ordre visuel. Le sonore, au contraire, emporte la forme, il ne la dissout pas, il l'élargit plutôt, il lui donne une ampleur, une épaisseur et une vibration ou une ondulation dont le dessin ne fait jamais qu'approcher. Le visuel persiste jusque dans son évanouissement, le sonore apparaît et disparaît jusque dans sa permanence.

Pourquoi et comment cette différence? Pourquoi et comment une ou plusieurs différence(s) des «sens » en général, et entre les sens sensibles et le sens sensé ? Pourquoi et comment quelque chose du sens sensé a privilégié un modèle, un support ou une référence dans la présence visuelle plutôt que dans la pénétration acoustique $^{10}$ ?

D'où viendrait cette « incapacité à dire le son et l'ouïe autrement qu'[...] en détournant, adaptant, trafiquant les concepts ou procédés nés des sciences liées à l'optique et à la $v^{11} e^{11} »$ ? Le son - ou l'écoute - en raison de son caractère plus discontinu et plus impermanent, brouille plus aisément les contours de toute interprétation herméneutique que l'on voudrait donner sans équivoque à un événement sensible. Il se présente au contraire à l'auditeur comme une énigme ouverte ou le « dénominateur commun de mondes, d'espaces sociaux au demeurant hétéroclites et souvent contradictoires $^{12} »$.

9 Accepter la part d'invisibilité du monde non pas comme une incomplétude peu rassurante mais comme un enrichissement de la perception et un catalyseur de nouveaux modes de relation et découverte de l'autre. Faire confiance au son non pas uniquement comme un moyen, un chemin pour arriver enfin à la source et la confirmation visuelle de son sens, mais, au contraire, le penser lui-même comme source et fleuve de sens mouvants et autonomes. Sens sensé qui ne peut jaillir (s'il jaillit) que du sens sensible. «Le son produit des sensations avant même de produire du sens. Et c'est par la sensation que le sens doit arriver ${ }^{13}$.»

Lors d'un banquet donné en son honneur par le syndicat français de la critique de cinéma, le 25 février 1982, dans les salons du Fouquet's à Paris, Orson Welles dit :

If the sound, and the rhythm of this sound - above all the rhythm - [are] wrong, no image can save it. [...] I believe sound is the first human sense for the theater, not the eye. I think the first theater was a story told by a storyteller. And the voice determines it. And the rhythm of the voice determines the images someway. It's not only what you say but the rhythm and speed of all the voices in the scene. So I often, when I direct, turn my back on the scene ${ }^{14}$. 
11 Tout son se fragmente en rythme. Tout rythme se traduit en son. Welles parle de son, de rythme, de tempo. C'est donc bien de musique qu'il parle. Non pas de la « discipline musique », mais de la musicalité au sens large, indissociable du phénomène théâtral et de la pratique théâtrale. La musique comme principe organisateur du temps dans l'espace et la succession, la superposition des couches sonores structurées en une durée qui, à défaut d'être immédiatement signifiante, trace un chemin ordonné dans le chaos des bruits du monde. « Le monde est bruit et silence. La musique extrait le son du bruit dans un sacrifice sanglant pour pouvoir articuler le bruit et le silence du monde ${ }^{15}$.»

12 C'est précisément cette articulation des couches sonores du monde, chaotique et harmonique tour à tour (ou à la fois), comme un vecteur de sens à part entière qu'il m'intéresse d'interroger dans mon travail à partir d'une donnée fondamentale du théâtre et qui ne cesse de revenir dans le discours de plusieurs praticiens : l'écoute. L'écoute est l'espace commun, le terrain d'entente partagé entre performeurs et spectateurs. Ni les uns ni les autres ne peuvent exister en dehors de cet espace d'écoute où des fils se tendent de performeur à performeur, de performeur à spectateur, de spectateur à spectateur formant une trame souple mais résistante qui sous-tend la dramaturgie ${ }^{16}$. À partir de ce "po(i)nt zéro", de cette écoute primordiale - qui se travaille et qui s'entretient quotidiennement - il s'agira d'interroger, par la pratique du plateau, les possibilités d'activation, croisement et mise en commun de matériaux sonores partagés dans l'espace poétique de la scène.

13 Comme son titre le laisse deviner, le projet de recherche Poétiques de la voix et espaces sonores que je mène est largement tributaire des réflexions de Paul Zumthor sur la vocalité, notamment là où elle se distingue de l'oralité et s'affranchit de la fonction de véhicule linguistico-sémantique, c'est-à-dire de transmetteur de sens. La musique de la langue, ou plutôt des langues, au-delà de toute intelligibilité immédiate, est naturellement convoquée à résonner dans cette grande composition chorale. Allemand, anglais, arabe, arménien, basque, bassa, batak, créole de Guadeloupe et d'Haïti, espagnol, filipino, flamand, fongbe, français, grec ancien et moderne, guarani, hébreu, hindi, indonésien, italien, japonais, kabyle, kongo, latin, lingala, mandarin, persan, portugais, roumain, russe, sanskrit, suédois, tamoul, ukrainien, yoruba..., trente-cinq langues sont entendues tout au long de ce spectacle qui n'est décidément pas fait pour être compris ( $\mathrm{du}$ moins pas intellectuellement), mais ressenti, touché, rappelé par l'écoute ${ }^{17}$.

\section{Chœurs cycliques}

14 Une des principales sources d'inspiration pour le dispositif de Théâtre fut l'installation sonore Forty Part Motet, créée en 2001 par l'artiste britannique Janet Cardiff ${ }^{18}$. Il s'agit d'un dispositif ellipsoïdal, formé par quarante enceintes montées sur piédestal, tournées vers le centre de l'ovale et divisées en huit groupes de cinq enceintes. Les espaces entre ces groupes permettent aux visiteurs d'aller et venir librement entre l'extérieur et l'intérieur de l'ellipse dessinée par les piédestaux. Chaque enceinte diffuse l'une des quarante voix du célèbre motet Spem in alium nunquam habui de Thomas Tallis - chef-d'œuvre de dentellerie contrapuntique composée en 1573 reconstituant, par la superposition simultanée des pistes, une polyphonie chorale. Le public se déplace à son gré à l'intérieur et à l'extérieur de l'espace circonscrit par cette 
ellipse. Le morceau, enregistré par le chœur de la cathédrale de Salisbury, est diffusé suivant une boucle de quatorze minutes, soit onze minutes de musique et trois minutes de pause. Le dispositif provoque une sensation d'écoute intime malgré son insertion dans un espace public ${ }^{19}$.

Notre idée fut donc de réinterpréter cette forme en la mettant en dialogue avec l'effet d'une présence humaine réelle, une performance dans l'ici et maintenant du théâtre, entendu principalement dans sa dimension relationnelle, c'est-à-dire, «ce qui se passe entre acteurs et spectateurs ${ }^{20} »$. Pas de décors, pas de costumes, pas d'éclairage, l'écoute seule est notre guide. Tout part d'elle et y revient. C'est à travers l'écoute que nous nous transformons et transformons l'espace. Cela nous paraissait être la forme idéale pour rendre compte, au théâtre, de la discontinuité sans cesse renouvelée, tour à tour globalisante et sélective, de l'écoute du monde, en une sorte d'écho à ce que dit Nancy à propos du sonore qui « apparaît et disparaît jusque dans sa permanence ».

Théâtre est donc un poème sonore polyphonique et polyglotte pour cinquante artistes en trente-cinq langues ${ }^{21}$, une promenade à la fois trompeuse et rassurante qui fait voler en éclats les repères spatio-temporels pour les reconstruire et déconstruire indéfiniment. Une trame de voix étalée dans un espace d'écoute. Des grands textes épiques, dramatiques ou poétiques en langue originale, ainsi que des musiques de différentes cultures deviennent des matériaux au même titre que des souvenirs, récits, témoignages, lettres, emails, messages vocaux ou écrits, journaux intimes apportés par les acteurs dans leurs langues respectives. Autant d'unités dramaturgiques pour une composition contrapuntique d'ensemble.

17 La plupart du temps, ces matériaux sont choisis et harmonisés dans le spectacle en fonction de critères essentiellement "musicaux »: contrastes rythmiques, musicalités similaires, ou parfois très opposées, qui se répondent, durées variées qui apportent constamment des dynamiques nouvelles à l'ensemble, textures, allitérations et assonances, complémentarité harmonique ou frottement dissonant de hauteurs ou " grains de voix ", emboîtements de sons et silences entre deux extraits sonores, entre autres.

Ainsi, par exemple, une polyphonie corse chantée par un chœur masculin qui dessine une diagonale traversant l'espace d'un bout à l'autre croise une lamentation en filipino parlée (ou pleurée) par une femme seule, immobile au centre du plateau. Un lied pour deux voix féminines et piano de Félix Mendelssohn dialogue avec le mot "arrête » répété plusieurs fois par une actrice dans une gradation d'états émotionnels qui vont du chuchotement affectueux à peine audible à la supplication désespérée à la limite du soutenable. Un trava-línguas (virelangue) en guarani, rythmé par un chœur masculin percussif, est progressivement "étouffé " par un chant grégorien qui transforme le chœur en basse continue sur un intervalle de quinte basses-ténors. Les dernières lignes de Finnegans Wake de James Joyce (en anglais) sont heurtées par des imprécations en fongbe, un chant traditionnel roumain, quelques notes d'une mélodie de Claude Debussy sur un poème de Paul Verlaine, le tout sous-tendu par un cluster choral sans cesse changeant. Deux personnages d'un épisode du Mahabharata, l'un parlant hindi et l'autre batak, se cherchent et s'évitent dans une dense forêt sonore d'où s'échappe aussi un chant en sanskrit extrait de la Bhagavad Gita...

Les fauteuils des spectateurs sont disposés en cercle et tournés vers l'espace laissé vide en son centre - il nous est aussi arrivé d'adopter, pour des questions de jauge et de dimensions du plateau, un dispositif avec deux cercles concentriques. Les spectateurs 
peuvent ainsi tous se voir les uns les autres immédiatement avant et après la performance, car pendant les quatre-vingt-cinq minutes que dure le spectacle, l'espace est plongé dans l'obscurité totale. La moindre source de lumière est en effet occultée et l'écoute devient le dénominateur commun, le fil d'Ariane des acteurs et des spectateurs.

Image $n^{\circ} 1$ - Théâtre, dispositif scénique sans les spectateurs.

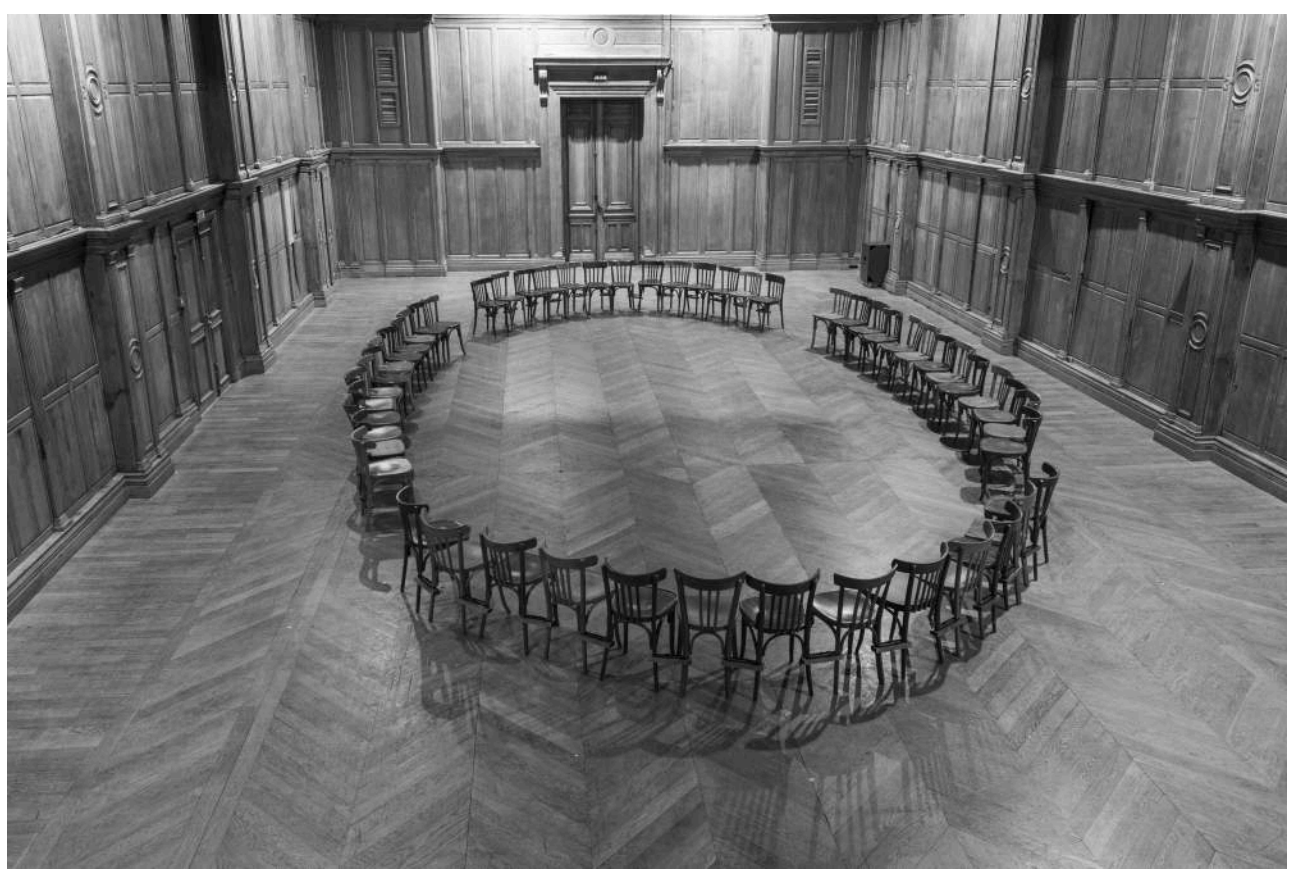

(c) Photo : Diego Bresani, avril 2015.

Image $n^{\circ} 2$ - Théâtre, dispositif scénique avec les spectateurs.

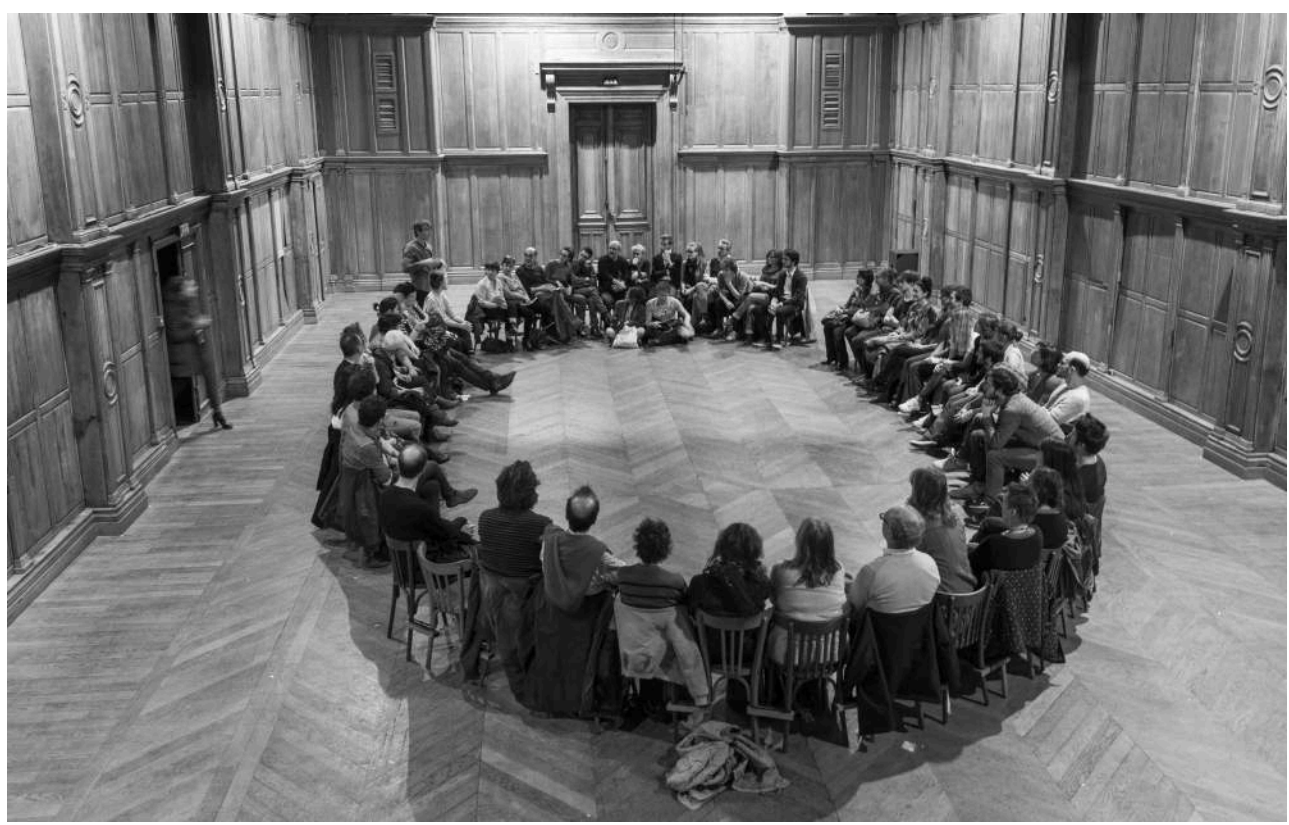

(c) Photo : Diego Bresani, avril 2015. 
premiers, entièrement nus, entrent en scène dans le noir - tout comme ils en sortiront - et forment à leur tour un cercle qui entoure celui formé par les derniers, en les plongeant dans un flux sonore ininterrompu aux multiples textures, couleurs, densités et dynamiques. Cet encerclement sera sans cesse relativisé et déstructuré tout au long du spectacle par des traversées, des éclatements et des tournoiements de voix dans les ténèbres. L'aire délimitée par le cercle se peuple et se dépeuple tour à tour, et nous faisons aussi jouer les angles de la salle, inscrivant, par le son, le cercle dans un carré ou un rectangle, redessinant constamment l'espace avec nos voix et les bruits de nos corps. Nous traçons des diagonales, nous croisons ou cassons des lignes, et nous jouons même avec la verticalité (et donc la hauteur, troisième dimension de notre "cage de scène ») en faisant monter une voix "dans les airs »: celle d'un acteuracrobate suspendu à un tissu que nous descendons et remontons dans le noir sans que le public s'en aperçoive. Sa voix monte progressivement, tourne dans les hauteurs avant de choir vertigineusement dans une triple vrille, déplaçant l'air qui frappe les spectateurs et réveille aussi leur sensibilité tactile.

Durant les dernières minutes du spectacle, les acteurs-performeurs se donnent enfin à voir (ou plutôt entrevoir) en s'éclairant eux-mêmes, l'un après l'autre, avec des petites lampes individuelles à faible puissance lumineuse. Alors, pour la première et dernière fois, la vision est sollicitée, et le public découvre le nombre et la nudité de celles et ceux qui l'ont entouré tout au long du parcours, en interaction les uns avec les autres, sans séparation scène-salle.

À ce moment précis, l'économie sonore change substantiellement. Si durant toute la performance les acteurs, plongés dans le noir, partageaient une invisibilité commune, ils se retrouvent à présent individualisés par la lumière. Ils produisaient auparavant un contrepoint complexe de chants, récits, déclamations, discussions, situations dramatiques, cris, rires, pleurs, chuchotements et imprécations d'où se détachaient par instant des "coryphées ", à présent ils font entendre tous, pour la première fois, la même note à l'unisson: un la médium. À l'uniformité de l'invisible se substitue l'uniformité sonore. Et la diversité visuelle succède à la diversité sonore. L'une et l'autre s'équilibrent et s'équivalent, l'une dévoilant le revers de l'autre. Il ne s'agit donc pas d'un chœur monolithique d'anonymes, mais au contraire, de la mise en commun des potentialités singulières de chacun. 
Image $n^{\circ} 3$ - Théâtre, minutes finales de la représentation avec acteurs (debout) placés tout autour du cercle des spectateurs (assis).

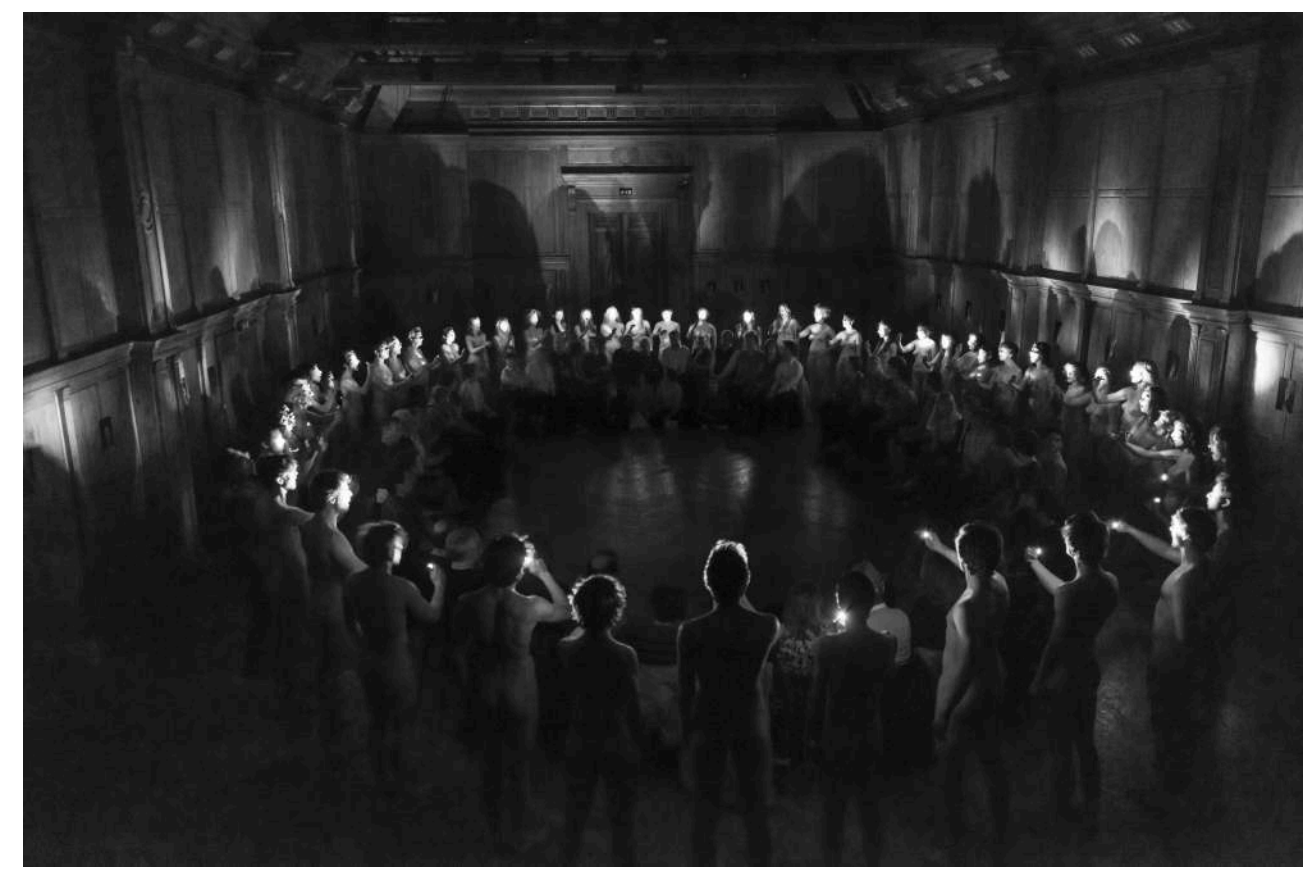

(C) Photo : Diego Bresani, avril 2015

23 Il faut en effet souligner que chaque acteur est appelé à composer une partie de la trame contrapuntique de Théâtre, en y apportant, sous forme de son, ses mémoires personnelles, en vocalisant ses propres racines (ou déracinements) culturelles et sociales. Âgés de 20 à 70 ans, les acteurs mettent en commun des matières vocales et des bagages sonores d'une richesse esthétique et humaine inestimable.

L'obscurité, qui renvoie tout autant à la solitude d'un espace très étroit qu'à la vastitude de l'infini, assure un mélange plus libre et plus perméable des couches tour à tour successives ou superposées de matières sonores plus ou moins détectables que nous déployons dans l'espace. Les multiples passés individuels s'entrecroisent et s'entrechoquent dans un présent partagé où la voix est un support catalyseur de mémoires intimes et collectives.

Nous avons pu vérifier, d'après les témoignages d'un grand nombre de spectateurs (et également d'acteurs) de ce spectacle, que le fait d'être plongé dans l'obscurité, exposé à un environnement sonore continu mais sans cesse changeant, déclenche des flux des perception inhabituels et des sensations alternées de profonde solitude et d'appartenance à une communauté en présence, de conscience aiguë de son propre corps et de dilution de ce dernier dans le corps collectif. Des souvenirs intimes enfouis émergent tour à tour pour disparaître aussitôt (et revenir peut-être) selon les circonstances acoustiques, sans cesse changeantes, auxquelles spectateurs et acteurs sont exposés.

Ce que l'écoute du monde me fait entendre, c'est une succession d'évènements qui, aussitôt appréciés, s'effacent dans ma mémoire. S'il existe un environnement, il est mnésique. [...] Le discontinu de l'oubli, aucun support ne peut en rendre compte. Pour commencer à pouvoir mettre en scène un espace sonore, il serait nécessaire de partir de la discontinuité, du surgissement, de l'évanouissement ${ }^{22}$. 


\section{Dramaturgies sonores} transformé par ce dernier. L'impression décrite par nombre de spectateurs, d'un «J'étais bien ici depuis tout ce temps?» ou d'un «C'est étrange, je n'arrive pas à retrouver le même espace où j'étais au début " ou encore d'un "C'était si petit que ça ?» suggère une contamination mnésique du visible par l'audible. Ces deux registres sont dissociés volontairement l'un de l'autre - à l'exception des trois minutes finales, ils ne partagent jamais le même espace-temps dans la structure dramaturgique. C'est la persistance de la mémoire d'un espace, projetée à l'intérieur d'un « nouvel » espace, qui modifie finalement les deux. Nous marquons cette transformation spatiotemporelle par le dévoilement d'un accrochage des portraits individuels de chacun des acteurs ${ }^{23}:$ une présence par l'absence ou une absence par la présence.

Image $\mathrm{n}^{\circ} 4$ - Théâtre, dispositif scénique après la représentation, avec accrochage des portraits.

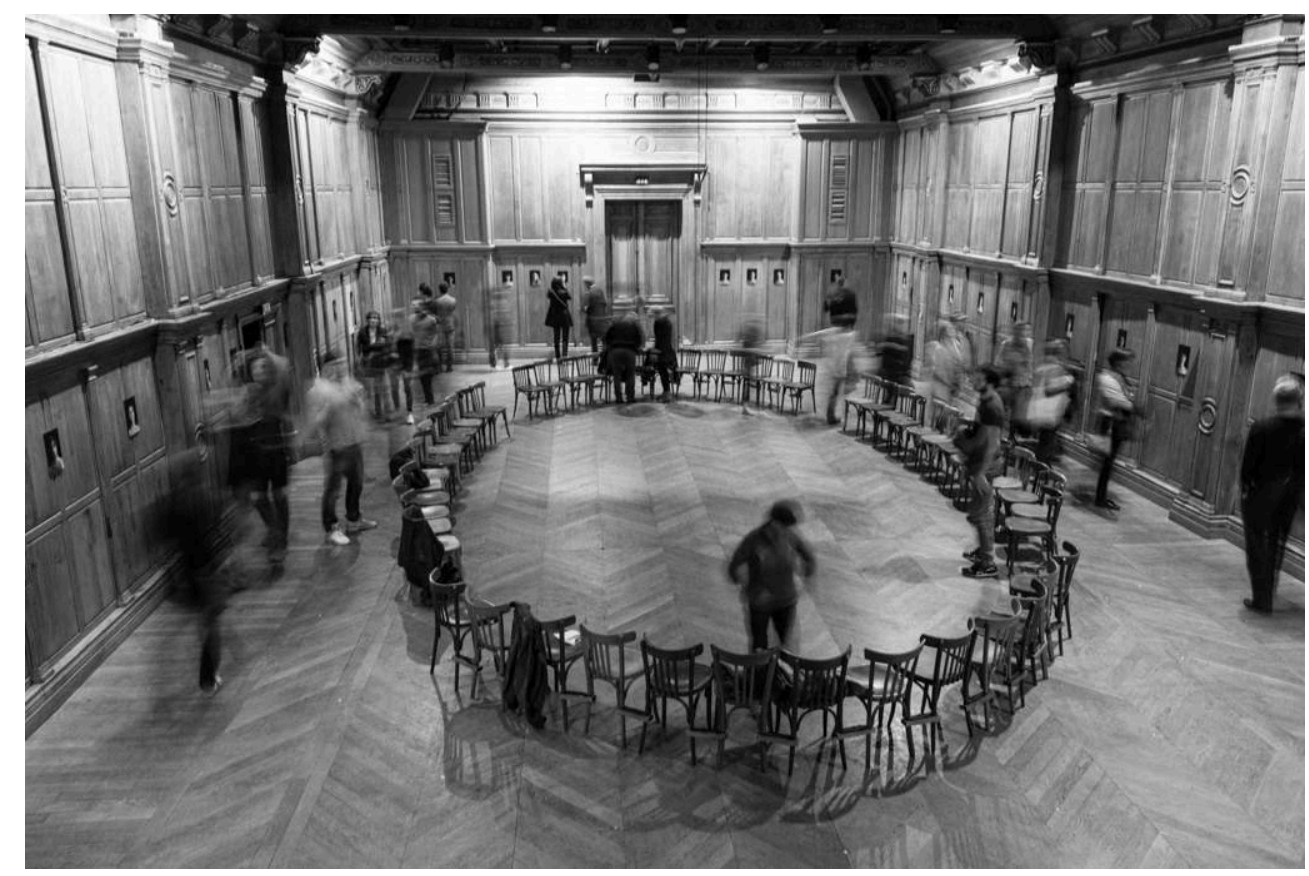

(c) Photo : Diego Bresani, avril 2015. 
une fois, l'image ne vient pas compléter ou confirmer la donnée sonore, car il est impossible d'identifier quelle voix entendue dans le noir appartient à quel visage muet, découvert sous la lumière. Pourtant, bien des spectateurs se prêtent à ce jeu et passent plusieurs minutes à scruter ces portraits offerts aux regards comme dans une galerie d'art (espace éminemment associé, bien plus que le théâtre, à la contemplation et au plaisir visuel). Les valeurs s'inversent donc : l'espace sonore du théâtre, créé par la rencontre présentielle d'acteurs et de spectateurs, fait place à l'espace visuel de l'exposition. Mais tout comme la mémoire de l'un est forcément contenue dans l'autre, le visuel peut émerger du sonore et vice versa. Si plusieurs spectateurs s'empressent de nous raconter toutes les images, les formes et les couleurs qu'ils ont vues (et ils insistent sur ce verbe) dans le noir, d'autres entendent encore des voix sortir de ces portraits « muets » dans l'espace visible déserté par les acteurs.

Ce qui nous intéressait dans le processus de Théâtre, ce n'était pas de monter une pièce dans le noir ou de raconter une histoire dont la voix acousmatique du narrateur déroulerait le fil (narratif) d'Ariane dans les ténèbres. Il ne s'agissait pas non plus de faire le bruitage d'une histoire invisible au moyen d'effets sonores illustratifs qui forceraient des images acoustiques anecdotiques et nécessairement tributaires de celles décrites « à la lettre » par la fable racontée. Tous ces procédés peuvent, certes, relever d'une recherche sonore complexe qui, dans certaines productions, atteint des degrés remarquables de raffinement mimétique dont l'exactitude hyperréaliste du dispositif sonore se substitue parfaitement à un récit visuel. Nous ne cherchions pas, justement, à substituer un sens à un autre, à mesurer le pouvoir pictural mimétique du son en un exercice de style consistant à raconter avec des bruits.

Il nous importait, au contraire, non seulement d'affranchir le sonore de sa position de «second»-après le visuel - dans la réception de l'événement scénique, mais surtout de l'affranchir de la fonction souvent réductrice de "bande son » à laquelle il est communément associé dans le montage d'un spectacle, c'est-à-dire au service d'une dramaturgie préalablement donnée, soit par le texte soit par la mise en scène, qu'il se doit de souligner ou d'illustrer sans qu'on s'en occupe comme un vecteur de sens à part entière ${ }^{24}$. Il s'agissait plutôt de penser une dramaturgie (et non pas une composition musicale ou un paysage sonore) à partir du son, des sons - aux natures, densités et fonctions très diverses - que nous avons produits et mis en commun tout au long du processus ${ }^{25}$.

31 Cette palette de possibilités acoustiques, nous l'avons restreinte aux sons corporels non transformés, amplifiés ou enregistrés, émis uniquement par le corps en mouvement et la vibration des cordes vocales ${ }^{26}$. L'organisation et l'harmonisation de ces sons dans l'espace-temps de la performance, faute d'un directeur de chœur visible, étaient possibles grâce à la mémoire phonique et l'écoute entraînée de l'ensemble des performeurs.

32 La forte présence de la choralité dans ce travail - de la salle de répétition, en tant qu'outil technique d'entraînement ${ }^{27}$, à la charpente du spectacle, en tant que socle structurant d'une dramaturgie fragmentaire et contrapuntique-, nous ne la revendiquons point comme métaphore de la communauté consensuelle (vouée à l'inertie) mais comme espace d'harmonisation dynamique des dissidences, des discordances, des dissonances, où l'être-ensemble n'implique pas une accumulation à somme nulle d'individus, mais un tissage en contrepoint de mémoires acoustiques et de réalités sonores. Le principe choral nous intéresse moins par l'agglomération que par la 
diversification génératrice de polyphonies. La musique au sens large, ou plutôt la musicalité comme principe organisateur des sons dans l'espace-temps, est ce qui nous permet de faire parler ensemble toutes ces voix et d'extraire, par intermittence, la symphonie de la cacophonie.

Partir de la voix et des croisements de voix dans leurs rapports avec le présent immédiat et (la mémoire de) l'écoute comme seuls garants de la composition dramaturgique, entretenue et renouvelée à chaque représentation, nous semblait un défi plus intéressant pour l'acteur, confronté à la double tâche de compositeur et d'interprète, assumée simultanément. D'autant plus que c'est bien du point de vue du théâtre que nous nous plaçons, c'est-à-dire, de la médiation performative d'une expérience relationnelle où acteurs et spectateurs partagent le même espace-temps. L'écoute isolée de toute autre contrainte ou référent extérieur nous confronte directement à cette vérité du théâtre selon laquelle il «réclame quotidiennement la réélaboration totale de son entité [...] sonore : aucune capitalisation autre que celle de la mémoire ${ }^{28} »$. Et c'est précisément de cela qu'il s'agit : proposer, à partir de l'élément sonore et de la vocalité, un espace-temps d'activation et de croisement de mondes sensibles, de mémoires sonores superposées et reconvoquées dans le présent de la scène sans l'intermédiaire du visuel.

\section{Présentation des extraits sonores}

Les documents audio proposés en prolongement de lecture de cet article ne rendent évidemment pas compte de tous les jeux de distance, profondeur, stratification et spatialisation sonores que ne peut ressentir qu'un spectateur placé au sein du dispositif scénique. Bien que quelques placements et déplacements d'acteurs ou groupes d'acteurs puissent être devinés à l'écoute - par le rapprochement ou l'éloignement des microphones placés en hauteur au centre du cercle - ces extraits ne sauraient remplacer l'expérience présentielle de cette forme où l'obscurité joue aussi un rôle capital dans la réception du spectacle. C'est plutôt comme des échantillons des différents matériaux sonores utilisés dans la construction de Théâtre que ces documents sont proposés en annexes.

Extrait $n^{\circ} 1$ - Captation de Théâtre (Conservatoire national supérieur d'art dramatique, 4 mars 2016).

Extrait $\mathrm{n}^{\circ} 2$ - Captation de Théâtre (Conservatoire national supérieur d'art dramatique, 4 mars 2016).

Extrait $\mathrm{n}^{\circ} 3$ - Captation de Théâtre (Conservatoire national supérieur d'art dramatique, 4 mars 2016).

Extrait $\mathrm{n}^{\circ} 4$ - Captation de Théâtre (Conservatoire national supérieur d'art dramatique, 4 mars 2016).

39 Il s'agit de quatre extraits de la représentation de Théâtre donnée le 4 mars 2016 à 15 heures dans la salle Louis Jouvet du Conservatoire national supérieur d'art dramatique à Paris. Les voix entendues dans cette captation sont celles de Jérôme Aubert, Roch Amedet Banzouzi, Sonia Belskaya, Marcus Borja, Augustin Bouchacourt, Lucie Brandsma, Sophie Canet, Antoine Cordier, Etienne Cottereau, Belén Cubilla, Mahshid Dastgheib, Alice Delagrave, Marcia Duarte, Simon Dusigne, Rachelle Flores, Michèle Frontil, François Gardeil, Lucas Gonzalez, Louise Guillaume, Lola Gutierrez, 
Jean Hostache, Hypo, Magdalena Ioannidi, Miléna Kartowski-Aïach, Matilda Kime, Cyrille Laik, Malec Lamraoui, Francis Lavainne, Feng Liu, Yuanye Lu, Hounhouénou Joël Lokossou, Esther Marty Kouyaté, Laurence Masliah, Jean-Max Mayer, Romane Meutelet, Tatiana Mironov, Makeda Monnet, Rolando Octavio, Ruchi Ranjan, Andrea Romano, Tristan Rothhut, Théo Salemkour, Charles Segard-Noirclère, Olivia Skoog, Aurore Soudieux, Isabelle Toros, Raluca Vallois, Gabriel Washer, Sophie Zafari, Vahram Zaryan.

\section{BIBLIOGRAPHIE}

Beaugrand, Claude, « Entendre l'arbre qui pousse », in R. La Rochelle (dir.), Écouter le cinéma, Montréal, 400 coups, 2002, p. 147-148.

Borja, Marcus, «L'écoute active et le silence parlant. La musicalité comme base pour la direction d'acteurs ", in J.-F. Dusigne (dir.), La Direction d'acteurs peut-elle s'apprendre ?, Paris, Les Solitaires intempestifs, 2015, p. 377-402.

Bovet, Jeanne, Larrue, Jean-Marc et Mervant-Roux, Marie-Madeleine, «Introduction », Voix Words Words Words, Théâtre/Public, nº 201, juil.-sept. 2011, p. 17-19.

Brook, Peter, L'Espace vide, Paris, Le Seuil, 1991.

Deshays, Daniel, « Le son du théâtre, un espace tactile », Le Son du théâtre, I. Le passé audible, Théâtre/Public, nº 197, oct. 2010, p. 20-23.

-, « Les mises en forme plastique et structurelle du sonore théâtral », Le Son du théâtre, II. Dire l'acoustique, Théâtre/Public, $\mathrm{n}^{\circ}$ 199, mars 2011, p. 46-47.

-, « Paysage sonore ?», in C. Guiu et al., (dir.), Soundspaces. Espaces, expériences et politiques du sonore, Rennes, PUR, « Géographie sociale », 2014, p. 25-30.

Foreman, Richard, «Le son, racine de mon théâtre », Voix Words Words Words, Théâtre/Public, $\mathrm{n}^{\circ} 201$, juil.-sept. 2011, p. 32-35.

Grotowski, Jerzy, Vers un théâtre pauvre, Lausanne, L'Âge d'Homme, 1993 [1971].

Nancy, Jean-Luc, À l'Écoute, Paris, Galilée, 2002.

Rancière, Jacques, Le Spectateur émancipé, Paris, La Fabrique, 2008.

Romieu, Patrick, « Désenchanter le sonore. Quelques considérations sur les méandres inférieurs de l'écoute ", in C. Guiu et al., (dir.), Soundspaces. Espaces, expériences et politiques du sonore, op. cit., p. 233-239.

Sterne, Jonathan, « Le passé audible », trad. fr. Françoise Ouellet, Le Son du théâtre, I. Le passé audible, Théâtre/Public, n ${ }^{\circ}$ 197, octobre 2010, p. 15-20.

Wisnik, José Miguel, o Som e o sentido, uma outra história das músicas [Le Son et le sens, une autre histoire des musiques], São Paulo, Companhia das Letras, 2011.

Zumthor, Paul, Introduction à la poésie orale, Paris, Le Seuil, 1983. 


\section{NOTES}

1. P. Zumthor, Introduction à la poésie orale, p. 11.

2. "J'étais sur le théâtre, en humeur d'écouter / La pièce, qu'à plusieurs j'avais ouï vanter » (Molière, Les Fâcheux, acte I, scène 1). Notons que, dans cet extrait, c'est bien le trait auditif, à savoir, l'action d'écouter, qui est associé à la " pratique du spectateur » devant la représentation théâtrale. Cependant, ne nous trompons pas : c'est bien à la pièce de théâtre en tant que texte écrit - comme il ne pouvait pas en être autrement dans la seconde moitié du XVII ${ }^{\mathrm{e}}$ siècle - que fait référence Éraste dans ce passage. C'est le texte de l'auteur qu'il lui importe d'écouter, à défaut de pouvoir (ou vouloir) le lire.

3. P. Brook, L'Espace vide, Paris, p. 19.

4. J. Rancière, Le Spectateur émancipé, p. 9.

5. Ibid., p. 10-11.

6. «Faire l'expérience, dans la représentation même, de ce qui est le fondement de l'expression, tout en empêchant l'attention de se focaliser sur la narration, sur le conflit entre les personnages ou sur une thématique. Le SON devient la dimension cruciale, inéluctable, étant donné que, contrairement au champ de vision, il ne peut pas être modifié par le spectateur rien qu'en fermant les yeux ou en brouillant la mise au point.» R. Foreman, «Le son, racine de mon théâtre »,p. 34 .

7. D. Deshays, « Le son du théâtre, un espace tactile », p. 21.

8. Théâtre fut créé les 7 et 8 avril 2015 dans la salle Louis Jouvet du Conservatoire national supérieur d'art dramatique et repris en 2016, les 17 et 18 février au Jeune théâtre national, dans le cadre du festival JT16, les 4 et 5 mars, à nouveau au CNSAD, et les 7 et 8 juin dans la grande salle de La Colline-Théâtre national dans le cadre du festival Impatience. Il a été programmé au Théâtre de la Cité internationale pour une dizaine de représentations du 24 au 28 avril 2017.

9. J. Sterne dénonce ce discours binaire d'oppositions étanches (et forcément réductrices) entre audition et vision qu'il appelle la « litanie audiovisuelle ». " La litanie décrit l'histoire des sens comme un jeu à "somme nulle", où la domination d'un sens mène nécessairement au déclin d'un autre. Cependant, l'affirmation selon laquelle l'utilisation d'un sens entraîne l'atrophie d'un autre n'est fondée sur aucune donnée scientifique », écrit-il dans «Le passé audible » (extrait de The Audible Past: Cultural Origins of Sound Reproduction, Durham, Duke University Press, 2003), traduit par Françoise Ouellet, p. 17. L'ouvrage original de l'auteur a fait ensuite l'objet d'une traduction intégrale: Une histoire de la modernité sonore, trad. fr. Maxime Boidy, Paris, La Découverte / Philharmonie de Paris, « La Rue musicale », 2015.

10. J.-L. Nancy, À l'écoute, p. 14.

11. " [1]'histoire et la théorie du théâtre n'ont pas échappé à la tentation générale. Elles parlent d'un monde autant auditif que visuel, mais ne disent à peu près rien de la première dimension, rien en tout cas qui soit à la mesure de l'expérience aurale de celui dont le nom même dit aujourd'hui l'éclipse sonore: "le spectateur". » (J.Bovet, J.-M. Larrue et M.-M. Mervant-Roux, «Introduction », p. 17).

12. P. Romieu, « Désenchanter le sonore. Quelques considérations sur les méandres inférieurs de l'écoute », p. 233.

13. D. Deshays, «Le son du théâtre, un espace tactile », p. 23.

14. Orson Welles déjeune avec la critique, émission Cinéma Cinémas, réalisée par Claude Ventura et Michel Boujut, diffusée sur Antenne 2 le 16 mars 1982, disponible sur le site de l'Institut national de l'audiovisuel à l'adresse suivante : http://www.ina.fr/video/I00008532

15. J. M. Wisnik, O Som e o sentido, uma outra história das músicas, p. 35 (ma traduction).

16. À ce propos, voir mon article «L'écoute active et le silence parlant. La musicalité comme base pour la direction d'acteurs », et en particulier, page 380 : « [e]lle [l'écoute] est perçue ici non pas - ou du moins pas uniquement - comme l'accomplissement et le résultat d'un mouvement de 
l'extérieur vers l'intérieur, un "se laisser traverser par", mais aussi - par opposition, et donc, dans une tension féconde et permanente - comme un mouvement de l'intérieur vers l'extérieur, une traversée active de l'espace et de l'autre. C'est précisément la tension créée entre ces deux mouvements qui génère un état de pleine présence [...] qui, même en l'absence de sons “audibles", rend même le silence palpable. »

17. Les langues listées ici sont celles de la reprise du spectacle dans la grande salle de La Colline lors du festival Impatience en juin 2016.

18. L'œuvre fait partie des collections permanentes de deux centres d'art: le musée des BeauxArts du Canada à Ottawa, où elle est exposée dans l'ancienne chapelle de la rue Rideau, depuis sa création en 2001, et l'Institut Inhotim à Brumadinho, au Brésil.

19. «While listening to a concert you are normally seated in front of the choir, in traditional audience position. With this piece I want the audience to be able to experience a piece of music from the viewpoint of the singers. Every performer hears a unique mix of the piece of music. Enabling the audience to move throughout the space allows them to be intimately connected with the voices. It also reveals the piece of music as a changing construct. As well I am interested in how sound may physically construct a space in a sculptural way and how a viewer may choose a path through this physical yet virtual space. » Propos de Janet Cardiff, consultables sur son site personnel : http://www.cardiffmiller.com/artworks/inst/motet.html

20. J. Grotowski, Vers un théâtre pauvre, p. 31.

21. Ce nombre, ainsi que la liste des langues, peut varier sensiblement d'un lieu à un autre et selon les changements de la distribution.

22. D. Deshays, « Paysage sonore ?», p. 30.

23. Cet accrochage est fait dans le noir tout au long du spectacle, sans que les spectateurs s'en aperçoivent. Quand les lumières sont rallumées à la fin, les portraits sont déjà en place.

24. «Engager une conception sonore au théâtre nécessite d'en questionner la place dès la première idée de mise en scène, dès le choix du texte ou de l'ébauche du projet. C'est dans un abord global à l'œuvre qu'il faut la penser. Or peu de metteurs en scène ont conscience du son et particulièrement de la place qui lui revient et qu'il faut lui attribuer pour lui permettre de s'insérer convenablement dans la création. Qui considère la voix dans son aspect sonore? Rappelons ici une fois encore que le son doit être conçu par le metteur en scène. ", D. Deshays, « Les mises en forme plastique et structurelle du sonore théâtral », p. 46.

25. «Pour cela, il faut modifier notre façon d'écouter, que l'oreille s'ajuste aux nouveaux objets sonores sans qu'il soit nécessaire de les justifier par l'image, ce qui est possible si ces sons se trouvent chargés d'un contenu émotionnel. Celui qui écoute recompose la trame sonore, lui prête des intentions et du sens. Il fabrique ses propres images en y injectant quelque chose de personnel: il participe à l'expérience ", C. Beaugrand, dans «Entendre l'arbre qui pousse ", p. 147-148.

26. Exception faite de l'utilisation ponctuelle d'instruments de musique - piano, accordéon, guitare, cajón et nyckelharpa (harpe à clés suédoise) - et aux deux montages sonores numériques d'environ deux minutes que nous utilisons au tout début et à la toute fin du spectacle pour les besoins de la mise en scène (masquer les bruits d'entrée et de sortie de scène des 50 acteurs). C'est pourtant à partir de la voix et dans une logique vocale que ces autres manifestations sonores viennent s'ajouter au tissu dramaturgique global.

27. Voir à ce propos M. Borja, art. cité.

28. D. Deshays, «Le son du théâtre, un espace tactile », p. 21. 


\section{RÉSUMÉS}

Jusqu'à quel point le théâtre est-il nécessairement dépendant de la vue ? L'élément acoustique est-il simplement le prolongement illustratif de l'élément visuel ? Peut-on aborder la création scénique à partir de sa dimension sonore? Telles sont les questions soulevées au sein de mon projet de recherche "Poétiques de la voix et espaces sonores", mené depuis 2014 dans le cadre du programme doctoral d'art et de création SACRe. Théâtre, pièce sonore chorale pour cinquante interprètes en trente-cinq langues, créée en avril 2015, en est la première restitution. Elle interroge les possibilités de construction de l'évènement scénique - aussi bien du point de vue dramaturgique que de celui de l'organisation des mouvements du plateau - uniquement à partir de matériaux sonores, et particulièrement vocaux. Il s'agit de composer, à travers l'écoute collective et la mémoire phonique, un tissage acoustique aux sens mouvants et multiples dans le présent partagé de la scène.

To what extent does the theatre necessarily depend upon sight? Is the acoustic element merely an illustrative extension of the visual element? Can we study scenic creation from its sonic dimension? Such are the questions raised within my research project, Poetics of the voice and sonic spaces » led since 2014 within the frame of SACRe, a doctoral program for art and creation, of which Théâtre, a sonic choral play for fifty players in thirty-five languages, produced in April 2015 , is the first emanation. The play interrogates the possibilities for constructing the scenic event - from the dramaturgic viewpoint as well as from the organization of stage movements only from sonic materials, and particularly vocal ones. What is at stake is the composition, through collective listening and vocal memory, of an acoustic weaving endowed with multiple, flexible meanings within the shared scenic present.

\section{INDEX}

Keywords : Sonic dramaturgy, listening, chorus, presence, voice

Mots-clés : dramaturgie sonore, écoute, chœur, présence, voix

\section{AUTEUR}

\section{MARCUS BORJA}

Docteur en études théâtrales (Sorbonne Paris Cité et Université de São Paulo), doctorant metteur en scène du programme SACRe («Science, Art, Création, Recherche »), École normale supérieure, Paris Sciences et Lettres et Conservatoire national supérieur d'art dramatique.

Parmi ses publications :

« Du collectif au collaboratif : tendances et évolutions de l'écriture scénique au pluriel », in R. Doyon et G. Freixe (dir.), « Du collectif au collaboratif : parcours de l'écriture scénique plurielle entre France et Brésil », Lavérune, L’Entretemps, « Les Points dans les poches », 2014, p. 169-188. «L'Écoute active et le silence parlant. La musicalité comme base pour la direction d'acteurs », in J.-F. Dusigne (dir.), La Direction d'acteurs peut-elle s'apprendre ?, Paris, Les Solitaires intempestifs, 2015, p. 377-402.

Poétiques de la voix sur scène, de l'apprentissage à la performance vocale, actes du colloque international des 16-18 novembre 2015, avec Ana Wegner et Chloé Larmet (dir.), Aix-enProvence, Presses universitaires de Provence, 2017 (à paraître). 